\title{
Eventos adversos em pacientes cirúrgicos: Revisão integrativa
}

\author{
Adverse events in surgical patients: An integrative review \\ Eventos adversos en pacientes quirúrgicos: Revisión integrativa
}

Recebido: 14/03/2021 | Revisado: 26/03/2021 | Aceito: 27/03/2021 | Publicado: 04/04/2021

\author{
Andressa dos Santos \\ ORCID: https://orcid.org/0000-0003-1648-9499 \\ Centro Universitário Santa Cruz de Curitiba, Brasil \\ E-mail: andressasantos509@yahoo.com.br \\ Natielen Pierobon \\ ORCID: https://orcid.org/0000-0001-6367-5513 \\ Centro Universitário Santa Cruz de Curitiba, Brasil \\ E-mail: natielenpierobom@gmail.com \\ Fernanda Aparecida Zarichen \\ ORCID: https://orcid.org/0000-0003-2650-8198 \\ Centro Universitário Santa Cruz de Curitiba, Brasil \\ E-mail: nandazari7@gmail.com \\ Gabriela Luísa Wibbelt \\ ORCID: https://orcid.org/0000-0002-3939-8904 \\ Centro Universitário Santa Cruz de Curitiba, Brasil \\ E-mail: gabrielawibbelto1@gmail.com \\ Ana Paula Moro Bertoni \\ ORCID: https://orcid.org/0000-0002-7987-1962 \\ Centro Universitário Santa Cruz de Curitiba, Brasil \\ E-mail: anapaulamoro.1980@gmail.com \\ Camila do Carmo Mota \\ ORCID: https://orcid.org/0000-0001-7140-4430 \\ Centro Universitário Santa Cruz de Curitiba, Brasil \\ E-mail: kmillacarmo@gmail.com \\ Luzia Souza Evangelista Pulcini \\ ORCID: https://orcid.org/0000-0002-2769-9654 \\ Centro Universitário Santa Cruz de Curitiba, Brasil \\ E-mail: luziasepulcini@yahoo.com.br \\ Silvelly Peres Teixeira \\ ORCID: https://orcid.org/0000-0001-7194-0102 \\ Centro Universitário Santa Cruz de Curitiba, Brasil \\ E-mail: silvelly16@gmail.com \\ Bruna Eloise Lenhani \\ ORCID: https://orcid.org/0000-0002-6009-3400 \\ Centro Universitário Santa Cruz de Curitiba, Brasil \\ E-mail: bruna.lenhani@unisantacruz.edu.br \\ Larissa Marcondes \\ ORCID: https://orcid.org/0000-0002-8745-6486 \\ Centro Universitário Santa Cruz de Curitiba, Brasil \\ E-mail: larissa.marcondes@unisantacruz.edu.br \\ Josemar Batista \\ ORCID: https://orcid.org/0000-0001-9838-1232 \\ Centro Universitário Santa Cruz de Curitiba, Brasil \\ E-mail: josemar.batista@unisantacruz.edu.br
}

\section{Resumo}

Objetivo: analisar as evidências científicas acerca dos eventos adversos ocorridos em pacientes cirúrgicos. Método: revisão integrativa de literatura, com coleta de dados de setembro a outubro de 2020, em três bases de dados: Literatura Latino-Americana e do Caribe em Ciências da Saúde (LILACS), Medical Literature Analysis and Retrieval System Online (MEDLINE) e Base de Dados em Enfermagem (BDENF). Foram selecionados artigos primários, publicados nos idiomas português, inglês e espanhol, sem recorte temporal. Resultados: foram elegíveis 18 artigos; 44,4\% ( $\mathrm{n}=8)$ foram conduzidos no Brasil. A amostra dos estudos variou de 19 a 11.949 registros/prontuários, com incidência/prevalência entre $3,5 \%$ a $21,8 \%$ e taxa de mortalidade estimada entre $1,82 \%$ a $18,4 \%$. A prevenção dos eventos variou de 7,7\% para casos relacionados à medicação a $90 \%$ para eventos gerais. Casos de eventos de origem infecciosa e de medicação/fluídos endovenosos foram prevalentes. Não seguir normas, falhas em habilidades técnicas, excesso de confiança e distrações foram fatores contribuintes para a ocorrência dos eventos adversos. Conclusão: a qualidade e a segurança do cuidado precisam ser aprimoradas em unidades de atendimento perioperatório, com destaque para os casos de infecção e de eventos adversos a medicamentos. 
Palavras-chave: Erros médicos; Procedimentos cirúrgicos operatórios; Segurança do paciente.

\begin{abstract}
Objective: to analyze the scientific evidences about adverse events occurred in surgical patients. Method: integrative literature review, with data collection from September to October 2020, in three databases: Latin American and Caribbean Literature on Health Sciences (LILACS), Medical Literature Analysis and Retrieval System Online (MEDLINE) and Nursing Database (BDENF). Primary articles were selected, published in Portuguese, English and Spanish, without temporal cutout. Results: 18 articles were eligible; 44,4\% $(n=8)$ were conducted in Brazil. The study sample ranged from 19 to 11.499 medical records, with incidence/prevalence of 3,5\% to $21,8 \%$, and estimated mortality rate of $1,82 \%$ to $18,4 \%$. The prevention of events ranged from $7,7 \%$ for cases related to medication to $90 \%$ for general events. Events associated with infection and medication/intravenous fluids were prevalent. Not following the norms, failure in technical skills, overconfidence and distractions were contributed factors to the occurrence of adverse events. Conclusion: the quality and safety of care need to be improved in perioperative care units, mainly in cases of infection and adverse drug events.
\end{abstract}

Keywords: Medical errors; Surgical procedures, operative; Patient safety.

\title{
Resumen
}

Objetivo: analizar evidencias científicas sobre eventos adversos en pacientes quirúrgicos. Método: revisión integradora de literatura, recolección de datos de septiembre a octubre de 2020, en tres bancos de datos: Literatura Latinoamericana y del Caribe en Ciencias de la Salud (LILACS), Medical Literature Analysis and Retrieval System Online (MEDLINE) y Base de Datos de Enfermería (BDENF). Se seleccionaron artículos primarios, publicados en portugués, inglés y español, sin marco temporal. Resultados: Se seleccionaron 18 artículos; El 44,4\% $(\mathrm{n}=8)$ realizados en Brasil. La muestra del estudio varió entre 19 y 11,949 registros/ficha médica, con incidencia/prevalencia entre $3,5 \%$ y $21,8 \%$ y tasa de mortalidad estimada entre $1,82 \%$ y $18,4 \%$. La prevención de eventos estuvo entre el $7,7 \%$ en casos relacionados con la medicación y el $90 \%$ para los eventos generales. Prevalecieron casos de eventos de origen infeccioso y por medicación/fluidos intravenosos. El incumplimiento de normas, fallas en habilidades técnicas, exceso de confianza y distracciones fueron factores que contribuyeron para que se presentaran eventos adversos. Conclusión: es necesario mejorar la calidad y seguridad de la atención en las unidades de cuidados perioperatorios, dándole especial importancia a los casos de infecciones y eventos adversos medicamentosos.

Palabras clave: Errores médicos; Procedimientos quirúrgicos operativos; Seguridad del paciente.

\section{Introdução}

A redução, a um mínimo aceitável, do risco de dano desnecessário compõe o conceito de segurança do paciente (SP) (Who, 2009). As discussões na temática se deram, majoritariamente, após a publicação do relatório do Institute of Medicine que divulgou elevados índices de incidentes de segurança relacionados aos ambientes de saúde (Kohn et al., 2000). Sabe-se que a SP é um dos seis atributos da qualidade do cuidado e tem adquirido importância para os pacientes, familiares, gestores e profissionais de saúde cuja finalidade é de oferecer assistência segura e de qualidade (Conass, 2017), com destaque para o ambiente cirúrgico por ser considerado área crítica e de maior risco assistencial ao paciente (Lopes et al., 2018).

Apesar da existência de limitações de acesso ao tratamento cirúrgico, estima-se que ocorram mundialmente 266 milhões de cirurgias ao ano (Bath et al., 2019). Esse dado suscita o desenvolvimento de condutas e estratégias para estimular boas práticas durante a atuação da equipe multiprofissional em diversos cenários de saúde, com vistas a reduzir os eventos adversos (EA). Esses são conceituados como incidentes que resultaram em danos ao paciente e representam elevada morbidade e mortalidade nos sistemas de saúde (Who, 2008; Who, 2009), principalmente se não identificados sinais e sintomas de alerta para ocorrência desses eventos pela equipe de enfermagem e demais profissionais da área (Breteler et al., 2020).

Nesse sentido, a Organização Mundial de Saúde (OMS) elencou, no biênio 2007-2008, o segundo desafio global em SP, no qual foi marcado pelo tópico "Cirurgias Seguras Salvam Vidas" e cujo objetivo é de reduzir a morbidade e mortalidade cirúrgica. (Who, 2008; Anvisa, 2017). Desde então, os Estado-Membros, incluindo o Brasil, vêm desenvolvendo e aprimorando estratégias para implementar a Lista de Verificação de Segurança Cirúrgica em seu formato de checklist, haja vista seus resultados promissores. Estudo norte-americano revelou que os hospitais que implantaram o uso dessa ferramenta reduziram em $22 \%$ os óbitos pós-cirúrgicos nos primeiros 30 dias (Haynes et al., 2017). 
Torna-se inegável os avanços ocorridos na SP cirúrgica. Entretanto, devido às desigualdades geográficas, garantir boas práticas nessa importante área assistencial persiste desafiante para gestores e profissionais de saúde ao considerar os diversos fatores que contribuem para promover cirurgias seguras, por exemplo, disponibilidade de materiais médicohospitalares e capacitação de equipes cirúrgicas (Moura et al., 2017). Ao identificar os EA e analisar seus fatores causais, torna-se possível entender de forma mais adequada acerca das falhas assistenciais com intuito de fornecer subsídios para promover cuidados seguros frente à adoção de ações exequíveis ao contexto investigado (Zanetti et al., 2020).

Desta forma, a presente pesquisa teve por objetivo analisar as evidências científicas acerca dos eventos adversos ocorridos em pacientes cirúrgicos.

\section{Metodologia}

Trata-se de uma revisão integrativa de literatura mediante a adoção de seis etapas (Mendes et al., 2008). A Etapa 1 foi composta pela identificação do problema e elaborada a questão de pesquisa: quais as evidências científicas acerca dos eventos adversos ocorridos em pacientes submetidos a procedimentos cirúrgicos?

A Etapa 2 consistiu na busca dos estudos primários em três bases de dados da Biblioteca Virtual de Saúde a saber: Literatura Latino-Americana e do Caribe em Ciências da Saúde (LILACS), Medical Literature Analysis and Retrieval System Online (MEDLINE) e Base de Dados em Enfermagem (BDENF). Essa etapa ocorreu no período compreendido de setembro a outubro de 2020 e utilizou-se o acrônimo PCC, em que a letra P - população; C - conceito e C - contexto. Para orientar a busca os descritores foram escolhidos conforme o acrômio PCC, Quadro 1.

Quadro 1 - Descritores para cada elemento da estratégia PCC - Curitiba, 2020.

\begin{tabular}{|l|l|}
\hline $\mathrm{P}=$ população & Paciente cirúrgico, procedimento cirúrgico operatório, assistência perioperatória. \\
\hline $\mathrm{C}=$ conceito & Erros médicos, eventos adversos \\
\hline $\mathrm{C}=$ contexto & Segurança do paciente, gerenciamento de segurança. \\
\hline
\end{tabular}

Fonte: Autores (2020).

Os descritores adotados para busca foram selecionados por meio do DeCS (Descritores em Ciências da Saúde), e também foram utilizados descritores não controlados. O Quadro 2 descreve as estratégias de busca. 
Quadro 2 - Estratégias de busca nas bases de dados - Curitiba, 2020.

\begin{tabular}{|c|l|c|}
\hline Bases de dados & \multicolumn{1}{|c|}{ Estratégia de busca } & n \\
\hline LILACS, BDENF e & "paciente cirúrgicos" $O R$ "procedimento cirúrgico operatório" $A N D$ "eventos adversos" & 18 \\
MEDLINE & $A N D$ "segurança do paciente" & 69 \\
\hline $\begin{array}{c}\text { LILACS, BDENF e } \\
\text { MEDLINE }\end{array}$ & $\begin{array}{l}\text { "paciente cirúrgicos" } O R \text { "procedimento cirúrgico operatório" } A N D \text { "erros médicos" } A N D \\
\text { "segurança do paciente" }\end{array}$ & 7 \\
MEDLINE & "eventos adversos" $A N D$ "pacientes cirúrgicos" $A N D$ "assistência perioperatória" & \\
\hline
\end{tabular}

Fonte: Autores (2020).

Adotaram-se os seguintes critérios de inclusão: artigos originais publicados eletronicamente na íntegra nos idiomas português, espanhol ou inglês; e que atendiam os itens do acrônimo PCC. Não foi adotado recorte temporal. Foram excluídos as teses, dissertações, relatos de casos e de experiência, editoriais, notas prévias e artigos de revisão.

A seleção dos estudos (Etapa 3) foi realizada por dois examinadores independentes mediante a leitura inicial dos títulos e resumos, destacando aqueles que respondiam à questão de pesquisa. Um terceiro examinador foi convidado a participar em casos de divergências em relação à inclusão do artigo para compor a fase subsequente. Após esse refinamento, procedeu-se às leituras recorrentes dos artigos na íntegra. Os casos elegíveis foram organizados e sumarizados em planilha do Microsoft Office Excel, versão 2016 (Etapa 4). As informações foram extraídas de acordo com instrumento validado (Ursi et al., 2006) e adaptado para o contexto da presente pesquisa a saber: autores, ano, país do estudo, periódico, título, objetivo, tipo de estudo/nível de evidência, fonte de dados e resultados.

O nível de evidência dos estudos foi classificado por meio da qualidade das evidências em sete níveis. As evidências provenientes de revisão sistemática ou meta-análise de todos relevantes ensaios clínicos randomizados controlados ou oriundas de diretrizes clínicas baseadas em revisões sistemáticas de ensaios clínicos randomizados controlados (Nível I), evidências derivadas de pelo menos um ensaio clínico randomizado controlado bem delineado (Nível II), evidências obtidas de ensaios clínicos bem delineados sem randomização (Nível III), evidências provenientes de estudos de coorte e de caso-controle bem delineados (Nível IV), evidências originárias de revisão sistemática de estudos descritivos e qualitativos (Nível V), evidências derivadas de um único estudo descritivo ou qualitativo (Nível VI), e evidências oriundas de opinião de autoridades e/ou relatório de comitês de especialistas (Nível VII) (Melnyk et al., 2005).

As variáveis foram apresentadas descritivamente em tabela, procedendo interpretações e comparações entre os resultados homogêneos e heterogêneos dos estudos selecionados para posterior apresentação da síntese do conhecimento (Etapas 5 e 6).

\section{Resultados}

Foram identificados 94 estudos pelas buscas nas bases de dados. Dos 23 artigos elegíveis, 18 compuseram a amostra final. O processo de seleção dos estudos está apresentado na Figura 1 de acordo com o Preferred Reporting Items for Systematic Review and a Meta-Analyses (PRISMA) (Galvão et al., 2015). 
Figura 1 - Fluxograma do processo de seleção do estudo.

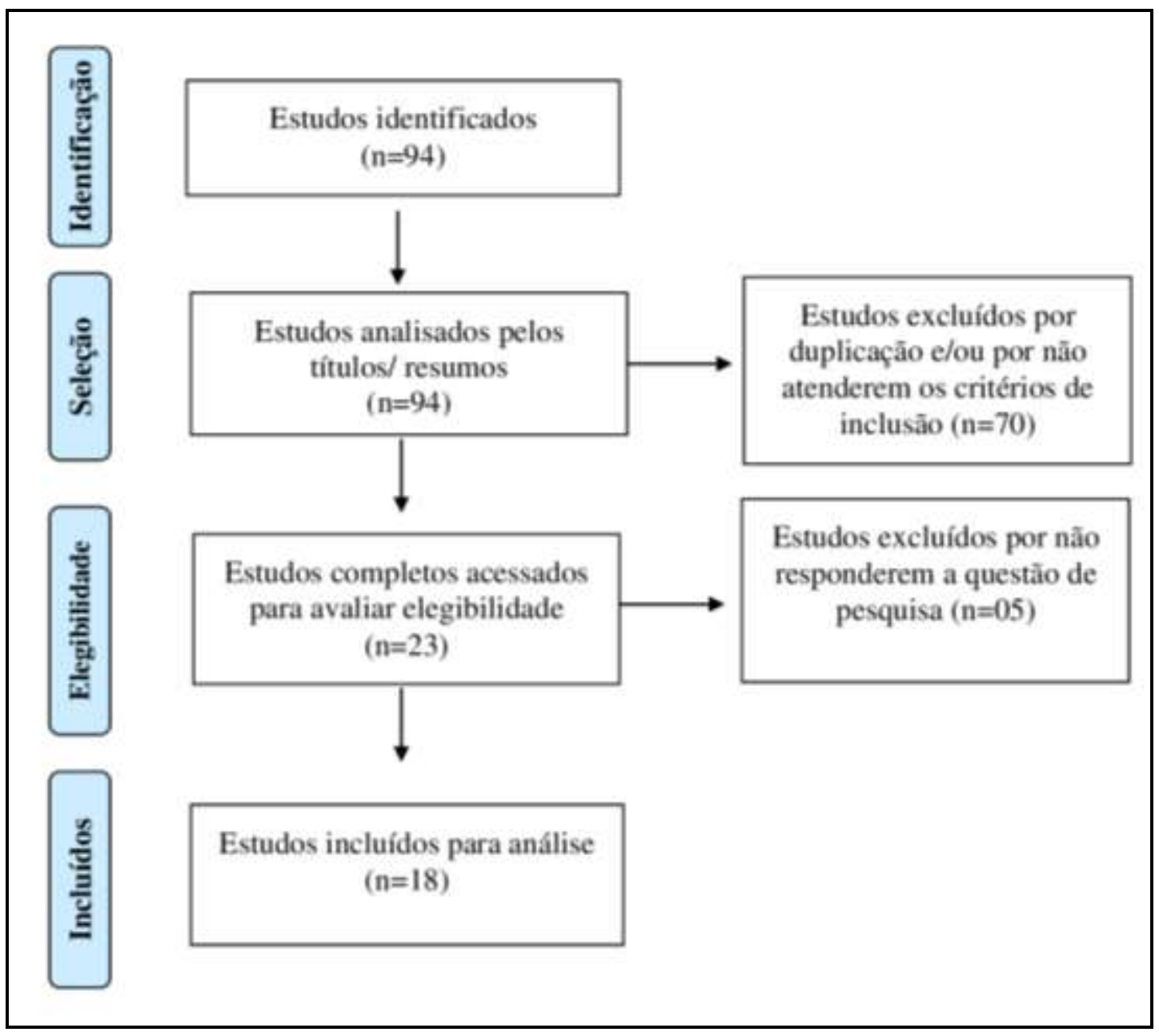

Fonte: Autores (2020).

Dentre os estudos selecionados, oito (44,4\%) foram conduzidos no Brasil (América do Sul), e quatro na América do Norte (22,2\%). Destes, uma investigação (5,6\%) foi multicêntrica. Cinco estudos (27,8\%) ocorreram no continente europeu, $5,6 \%(\mathrm{n}=1)$ no continente asiático. Não houve publicações no período compreendido de 2007 a 2010, e 55,56\%(n=10) dos artigos foram publicados há menos de cinco anos, com destaque para o ano de 2018, com três publicações (16,6\%).

Foram identificadas 14 produções com nível de evidência VI (77,77\%), e majoritariamente, os estudos foram desenvolvidos com delineamento retrospectivo, com análise em prontuários ou livros de registros da enfermagem $(\mathrm{n}=14$; $77,78 \%$ ), com amostra variando de 19 a 11.949 registros/prontuários.

O Quadro 3 descreve os estudos conforme autoria, ano de publicação, periódico, título, objetivo, delineamento metodológico, população/amostra, nível de evidência, incidência/prevalência, prevenção e grau de dano dos EA ocorridos em pacientes submetidos a procedimentos anestésicos e cirúrgicos. 
Quadro 1 - Síntese dos artigos incluídos na revisão. Curitiba, PR, Brasil, 2020 (n=18).

\begin{tabular}{|c|c|c|c|c|c|}
\hline $\begin{array}{l}\text { Autoria/Ano/ } \\
\text { País do } \\
\text { estudo/ } \\
\text { Periódico }\end{array}$ & 'ítulo & Objetivo & $\begin{array}{c}\text { Tipo de } \\
\text { estudo/ Nível } \\
\text { de evidência }\end{array}$ & $\begin{array}{c}\text { Período de } \\
\text { análise/Número de } \\
\text { prontuários/Registros } \\
\text { amostrados }\end{array}$ & Principais destaques \\
\hline $\begin{array}{l}\text { Medina-Franco } \\
\text { et al., 2006, } \\
\text { México. }\end{array}$ & $\begin{array}{l}\text { Factores } \\
\text { asociados a } \\
\text { mortalidad en } \\
\text { cirugía mayor: } \\
\text { análisis } \\
\text { retrospectivo en } \\
\text { un centro de } \\
\text { referencia }\end{array}$ & $\begin{array}{l}\text { Estudar as causas e } \\
\text { os fatores } \\
\text { associados à } \\
\text { mortalidade } \\
\text { cirúrgica. }\end{array}$ & $\begin{array}{l}\text { Transversal e } \\
\text { retrospectivo/ } \\
\text { VI }\end{array}$ & $\begin{array}{l}4.157 \text { procedimentos } \\
\text { cirúrgicos realizados em } \\
\text { um centro de referência } \\
\text { terciário no ano de } 2000 .\end{array}$ & $\begin{array}{l}\text { A taxa de mortalidade foi de } \\
1,82 \% \text {. Em } 33,8 \% \text { dos óbitos } \\
\text { pós-cirúrgicos, um EA foi } \\
\text { identificado como responsável } \\
\text { pelo desfecho. Em } 23 \% \text { dos } \\
\text { casos houve um evento } \\
\text { potencialmente } \\
\text { representando evitável, } \\
\text { procedimentos cirúrgicos. A } \\
\text { infecção de sitio cirúrgico } \\
\text { associada a sepse foi a } \\
\text { complicação mais frequente. }\end{array}$ \\
\hline $\begin{array}{l}\text { Souza et al., } \\
\text { 2011, Brasil. } \\
\text { Rev. enferm. } \\
\text { UERJ }\end{array}$ & $\begin{array}{l}\text { Eventos } \\
\text { adversos: } \\
\text { instrumento de } \\
\text { avaliação do } \\
\text { desempenho } \\
\text { em centro } \\
\text { cirúrgico de um } \\
\text { hospital } \\
\text { universitário }\end{array}$ & $\begin{array}{l}\text { Caracterizar os EA } \\
\text { ocorridos na } \\
\text { unidade de centro } \\
\text { cirúrgico de um } \\
\text { hospital } \\
\text { universitário da } \\
\text { Região Centro- } \\
\text { Oeste do Brasil }\end{array}$ & $\begin{array}{l}\text { Documental e } \\
\text { retrospectivo/ } \\
\text { VI }\end{array}$ & $\begin{array}{l}\text { Todos os registros nos } \\
\text { livros de anotações de } \\
\text { Enfermagem no período } \\
\text { compreendido de } 2005 \text { a } \\
2009 \text {. }\end{array}$ & $\begin{array}{l}\text { Foram identificados } 42 \text { EA. Dos } \\
31 \text { EA relacionados à } \\
\text { assistência, observou-se que } \\
35,5 \% \text { foram oriundos do ato } \\
\text { anestésico. Outros } 7 \text { (22,6\%) } \\
\text { EA se relacionavam à parada } \\
\text { cardiorrespiratória e ao } \\
\text { pneumotórax. }\end{array}$ \\
\hline $\begin{array}{l}\text { Carneiro et al., } \\
\text { 2011, Brasil. }\end{array}$ & $\begin{array}{l}\text { Eventos } \\
\text { adversos na } \\
\text { clínica cirúrgica } \\
\text { de um hospital } \\
\text { universitário: } \\
\text { instrumento de } \\
\text { avaliação da } \\
\text { qualidade }\end{array}$ & $\begin{array}{l}\text { Analisar os EA } \\
\text { ocorridos na } \\
\text { clínica cirúrgica de } \\
\text { um hospital } \\
\text { universitário } \\
\text { pertencente à rede } \\
\text { de hospitais } \\
\text { sentinela. }\end{array}$ & $\begin{array}{l}\text { Documental e } \\
\text { retrospectivo/ } \\
\text { VI }\end{array}$ & $\begin{array}{llr}\text { Todos } & \text { os } & \text { registros } \\
\text { contidos } & \text { no livro de } \\
\text { anotações } & \text { do enfermeiro } \\
\text { entre os } & \text { anos } 2005 \text { e } \\
2009 & & \end{array}$ & $\begin{array}{l}\text { Foram encontrados } 264 \text { EA. } \\
\text { Houve prevalência de } \\
\text { relacionados a retirada de } \\
\text { sondas, drenos e cateteres } \\
(61,36 \%) \text {, quedas }(18,56 \%) \text {, } \\
\text { reações alérgicas a } \\
\text { medicamentos (5,30\%), evasão } \\
(5,30 \%) \text {, lesão por pressão } \\
(4,92 \%) \text {. Em } 46,21 \% \text { dos casos, } \\
\text { constatou-se dano temporário } \\
\text { com necessidade } \\
\text { intervenção. }\end{array}$ \\
\hline $\begin{array}{l}\text { Moura et al., } \\
\text { 2012, Brasil. }\end{array}$ & $\begin{array}{l}\text { Avaliação de } \\
\text { eventos } \\
\text { adversos } \\
\text { cirúrgicos em } \\
\text { hospitais do Rio }\end{array}$ & $\begin{array}{lr}\text { Avaliar } & \text { a } \\
\text { incidência de } & \text { EA } \\
\text { cirúrgicos e } & \text { os } \\
\text { fatores } & \\
\text { contributivos } & \text { em }\end{array}$ & $\begin{array}{l}\text { Coorte } \\
\text { retrospectivo/ } \\
\text { IV }\end{array}$ & $\begin{array}{l}1.103 \text { prontuários de } \\
\text { pacientes internados em } \\
2003 \mathrm{em} \text { três hospitais } \\
\text { públicos. }\end{array}$ & $\begin{array}{l}\text { A incidência foi de } 3,5 \% \text {. Dos } \\
41 \text { EA, } 68,3 \% \text { eram evitáveis. A } \\
\text { proporção de pacientes } \\
\text { submetidos à cirurgia com EA } \\
\text { cirúrgicos foi } 5,9 \% \text {. Dentre os }\end{array}$ \\
\hline
\end{tabular}


Research, Society and Development, v. 10, n. 4, e16810413896, 2021

(CC BY 4.0) | ISSN 2525-3409 | DOI: http://dx.doi.org/10.33448/rsd-v10i4.13896

\begin{tabular}{|c|c|c|c|c|c|}
\hline epidemiol & de Janeiro. & $\begin{array}{l}\text { hospitais do Rio de } \\
\text { Janeiro. }\end{array}$ & & & $\begin{array}{l}\text { pacientes com EA cirúrgico, } 3 \\
(7,9 \%) \text { tiveram mais de um EA, } \\
\text { uma média de } 1,1 \text { eventos por } \\
\text { paciente. A taxa de mortalidade } \\
\text { foi estimada em } 18,4 \% \text {. Nove } \\
\text { pacientes (21,9\%) tiveram } \\
\text { danos permanentes, dos quais } 7 \\
(17,1 \%) \text { foram óbitos. Houve } \\
\text { prevalência de EA relacionados } \\
\text { a ferida cirúrgica, sendo } 19,5 \% \\
\text { por infecção e } 26,8 \% \text { por outro } \\
\text { problema com a ferida cirúrgica. } \\
\text { O fator norma - não verificar ou } \\
\text { não seguir protocolo contribuiu } \\
\text { em } 31,0 \% \text { dos eventos e } \\
\text { habilidade - erros ou descuidos } \\
\text { em } 14,3 \% \text { dos casos analisados. }\end{array}$ \\
\hline $\begin{array}{l}\text { Paranaguá et } \\
\text { al., } \\
\text { 2013, Brasil. } \\
\text { Acta paul. } \\
\text { enferm }\end{array}$ & $\begin{array}{l}\text { Prevalência de } \\
\text { incidentes sem } \\
\text { dano e eventos } \\
\text { adversos em } \\
\text { uma clínica } \\
\text { cirúrgica }\end{array}$ & $\begin{array}{l}\text { Estimar a } \\
\text { prevalência de } \\
\text { incidentes sem } \\
\text { dano e EA em uma } \\
\text { clínica cirúrgica. }\end{array}$ & $\begin{array}{l}\text { Transversal e } \\
\text { retrospectivo/ } \\
\text { VI }\end{array}$ & $\begin{array}{l}750 \text { prontuários de } \\
\text { pacientes internados no } \\
\text { ano de } 2010 \text { em uma } \\
\text { clínica cirúrgica. }\end{array}$ & $\begin{array}{l}\text { Constatou-se prevalência de EA } \\
\text { de } 18,7 \% \text {. Dos } 218 \text { casos, os } \\
\text { mais recorrentes foram } \\
\text { relacionados ao processo } \\
\text { clínico, com destaque para a dor } \\
\text { aguda em pós-operatório } \\
\text { (n=54;24,77\%). Retirada não } \\
\text { programada de sonda/dreno } \\
\text { contribuiu com } 23 \text { casos } \\
(10,60 \%) \text { seguida de falhas de } \\
\text { procedimentos técnicos } \\
\text { (n=13;5,96\%), obstrução de } \\
\text { cateter/sonda/tubo traqueal } \\
(n=13 ; 5,96 \%) \text { e deiscência } \\
\text { cirúrgica com oito casos } \\
(3,67 \%) \text {. }\end{array}$ \\
\hline $\begin{array}{l}\text { Baines et al., } \\
\text { 2013, Holanda } \\
\text { BMJ Qual Saf. }\end{array}$ & $\begin{array}{lr}\text { Changes in } \\
\text { adverse event } \\
\text { rates in } \\
\text { hospitals over } \\
\text { time: } \quad \text { a } \\
\text { longitudinal } \\
\text { retrospective } \\
\text { patient record } \\
\text { review study. }\end{array}$ & $\begin{array}{l}\text { Determinar } \\
\text { alternações nas } \\
\text { taxas de EA, } \\
\text { identificar grupos } \\
\text { de risco de } \\
\text { pacientes e discutir } \\
\text { os fatores que } \\
\text { influenciam } \\
\text { resultado. }\end{array}$ & $\begin{array}{l}\text { Longitudinal/ } \\
\text { IV }\end{array}$ & $\begin{array}{l}\text { Amostra aleatória de } 21 \\
\text { hospitais em } 2004 \text { e } 20 \\
\text { hospitais em 2008. Para } \\
\text { cada hospital foram } \\
\text { incluídas } 400 \text { internações } \\
\text { de pacientes no ano de } \\
2004 \text { e } 200 \text { internações } \\
\text { em } 2008 \text {. Foram } \\
\text { revisados } \\
\text { prontuários. }\end{array}$ & $\begin{array}{l}\text { A taxa de pacientes com EA } \\
\text { aumentou de } 4,1 \text { para } 6,2 \% \text { em } \\
2008 \text {, enquanto que a taxa de } \\
\text { EA evitável permaneceu estável } \\
\text { em } 1,8 \% \quad(2008) \text {. Foram } \\
\text { detectados } 516 \text { EA cirúrgicos } \\
(51,7 \%) ; 36,5 \% \text { evitáveis. }\end{array}$ \\
\hline
\end{tabular}




\begin{tabular}{|c|c|c|c|c|c|}
\hline $\begin{array}{l}\text { Boer et al., } \\
\text { 2013, Holanda. } \\
\text { J Eval Clin } \\
\text { Pract }\end{array}$ & $\begin{array}{l}\text { A targeted } \\
\text { method for } \\
\text { standardized } \\
\text { assessment of } \\
\text { adverse } \\
\text { drug events in } \\
\text { surgical } \\
\text { patients }\end{array}$ & $\begin{array}{l}\text { Demonstrar o } \\
\text { desenvolvimento, } \\
\text { confiabilidade e } \\
\text { resultado de um } \\
\text { método } \\
\text { direcionado para } \\
\text { avaliação } \\
\text { padronizada de EA } \\
\text { a medicamentos } \\
\text { (EAM) } \\
\text { pacientes } \\
\text { cirúrgicos. }\end{array}$ & $\begin{array}{l}\text { Coorte } \\
\text { prospectivo/ IV }\end{array}$ & $\begin{array}{l}262 \text { pacientes com } \\
\text { admissão superior a } 48 \\
\text { horas e com internação } \\
\text { ocorrida entre março e } \\
\text { junho de } 2009 \text { em três } \\
\text { enfermarias cirúrgicas. }\end{array}$ & $\begin{array}{l}\text { Foram encontrados } 91 \text { EAM em } \\
76 \text { pacientes; } 7,7 \% \text { eram } \\
\text { evitáveis. Em } 73,6 \% \text { dos EAM } \\
\text { estiveram relacionados a } \\
\text { medicamentos de uso para o } \\
\text { sistema nervoso como os } \\
\text { opióides. Dos casos evitáveis, } \\
28,6 \% \text { foram classificados de } \\
\text { graves a potencialmente fatais. }\end{array}$ \\
\hline $\begin{array}{l}\text { Wang et al., } \\
\text { 2014, EUA. }\end{array}$ & $\begin{array}{l}\text { National Trends } \\
\text { in Patient } \\
\text { Safety for Four } \\
\text { Common } \\
\text { Conditions, } \\
2005-2011\end{array}$ & $\begin{array}{l}\text { Avaliar a tendência } \\
\text { nas taxas de EA } \\
\text { entre pacientes } \\
\text { hospitalizados por } \\
\text { infarto agudo do } \\
\text { miocárdio, } \\
\text { insuficiência } \\
\text { cardíaca } \\
\text { congestiva, } \\
\text { pneumonia ou } \\
\text { condições que } \\
\text { requerem cirurgia. }\end{array}$ & $\begin{array}{l}\text { Transversal } \\
\text { analítico/ VI }\end{array}$ & $\begin{array}{l}\text { Dados de } 16.481 \\
\text { internações ocorridas no } \\
\text { período de } 2005 \text { a } 2011 . \\
\text { Participaram da pesquisa } \\
4.372 \text { hospitais. }\end{array}$ & $\begin{array}{l}\text { Não houve diminuição } \\
\text { significativa nas taxas de EA } \\
\text { em pacientes cirúrgicos. O } \\
\text { número de eventos passou de } \\
352,3 \text { (2005-2006) para 368,1 } \\
\text { por } 1.000 \text { hospitalizações em } \\
2010-2011 \text {. Na série temporal } \\
\text { (2010-2011) houve prevalência } \\
\text { de eventos relacionados a } \\
\text { administração de heparina } \\
\text { endovenosa (32\%), seguido de } \\
\text { nefropatia após angiografia com } \\
\text { contraste (29,3\%), pneumonia } \\
\text { associada a ventilação mecânica } \\
(10,6 \% \text { e lesão por pressão } \\
(6,4 \%) \text {. }\end{array}$ \\
\hline $\begin{array}{l}\text { Silva et al., } \\
\text { 2015, Brasil. } \\
\text { Rev. SOBECC }\end{array}$ & $\begin{array}{l}\text { Análise de } \\
\text { eventos } \\
\text { adversos em um } \\
\text { centro cirúrgico } \\
\text { ambulatorial }\end{array}$ & $\begin{array}{l}\text { Analisar os } \text { EA } \\
\text { notificados no } \\
\text { Centro Cirúrgico } \\
\text { para a segurança } \\
\text { do paciente }\end{array}$ & $\begin{array}{l}\text { Documental e } \\
\text { retrospectivo/ } \\
\text { VI }\end{array}$ & $\begin{array}{l}\text { Banco de dados do setor, } \\
\text { com os } 250 \text { casos } \\
\text { notificados em sistema } \\
\text { institucional no ano de } \\
2014 .\end{array}$ & $\begin{array}{l}\text { Foram notificados } 17 \text { EA } \\
(19 \%) \text {. Sete }(7,8 \%) \text { estiveram } \\
\text { relacionados ao próprio } \\
\text { paciente, com destaque para } \\
\text { queimaduras ocasionadas pelo } \\
\text { eletrocautério. Erro de } \\
\text { medicação representou } 5,6 \% \text {, } \\
\text { seguido de reação adversa } \\
(2,2 \%) \text {, flebite, queda e lesão } \\
\text { por pressão com } 1,1 \% \text { para cada } \\
\text { evento. }\end{array}$ \\
\hline
\end{tabular}




\begin{tabular}{|c|c|c|c|c|c|}
\hline $\begin{array}{l}\text { Thiels et al., } \\
\text { 2015, EUA }\end{array}$ & $\begin{array}{l}\text { Surgical never } \\
\text { events and } \\
\text { contributing } \\
\text { human factors }\end{array}$ & $\begin{array}{l}\text { Analisar os fatores } \\
\text { que contribuem } \\
\text { para ocorrência nos } \\
\text { never events }\end{array}$ & $\begin{array}{l}\text { Estudo } \\
\text { transversal/ VI }\end{array}$ & $\begin{array}{l}\text { Banco de dados contendo } \\
\text { informações dos } \\
\text { incidentes ocorridos entre } \\
31 \text { de agosto de } 2009 \text { a } \\
31 \text { de agosto de } 2014 \text { de } \\
\text { um hospital terciário. }\end{array}$ & $\begin{array}{l}\text { Foram encontrados } 70 \text { never } \\
\text { events, com destaque para } \\
\text { objetos retidos inadvertidamente } \\
\text { e colocação de implantes } \\
\text { errados, os quais exigiram } \\
\text { operação ou procedimento } \\
\text { adicional em } 75 \% \text { e } 60 \% \text {, } \\
\text { respectivamente. A taxa de } \\
\text { reoperação foi de } 45 \% \text { para } \\
\text { procedimento errado e de } 42 \% \\
\text { para cirurgia no lado/local } \\
\text { errado. Fatores cognitivos, por } \\
\text { exemplo, excesso de confiança e } \\
\text { distrações contribuíram para a } \\
\text { ocorrência da maioria dos casos. }\end{array}$ \\
\hline $\begin{array}{l}\text { Sell et al., } \\
\text { 2016, Brasil. } \\
\text { Rev. SOBECC }\end{array}$ & $\begin{array}{l}\text { Eventos } \\
\text { adversos em } \\
\text { uma unidade de } \\
\text { internação } \\
\text { cirúrgica: } \\
\text { estudo } \\
\text { descritivo }\end{array}$ & $\begin{array}{l}\text { Verificar } \\
\text { ocorrência de } \\
\text { infecção do sítio } \\
\text { cirúrgico, perda ou } \\
\text { infecção do acesso } \\
\text { venoso e quedas } \\
\text { em pacientes } \\
\text { internados em } \\
\text { Unidade de } \\
\text { Internação } \\
\text { Cirúrgica de um } \\
\text { hospital de ensino. }\end{array}$ & $\begin{array}{l}\text { Descritivo e } \\
\text { quantitativo/VI }\end{array}$ & $\begin{array}{l}94 \text { pacientes internados } \\
\text { no ano de } 2013, \\
\text { totalizando } \\
\text { avaliações. }\end{array}$ & $\begin{array}{l}\text { Observou-se um total de } 20 \text { EA } \\
(7,9 \%) \text {. Nove notificações } \\
\text { estavam relacionadas a perda de } \\
\text { acesso venoso }(3,6 \%) \text {, sete } \\
\text { acerca de infecção de sitio } \\
\text { cirúrgico }(2,8 \%) \text {, e quatro casos } \\
\text { notificados eram de infecção em } \\
\text { acesso venoso }(1,6 \%) .\end{array}$ \\
\hline $\begin{array}{l}\text { Halfon et al., } \\
\text { 2017, Suíça } \\
\text { Int J Qual } \\
\text { Health Care }\end{array}$ & $\begin{array}{l}\text { Adverse events } \\
\text { related to } \\
\text { hospital care: a } \\
\text { retrospective } \\
\text { medical records } \\
\text { review in a } \\
\text { Swiss hospital }\end{array}$ & $\begin{array}{l}\text { Estimar a } \\
\text { incidência de EA. }\end{array}$ & $\begin{array}{l}\text { Transversal e } \\
\text { retrospectivo / } \\
\text { VI }\end{array}$ & $\begin{array}{l}\text { Amostra estratificada de } \\
400 \quad \text { hospitalizações } \\
\text { cirúrgicas e } 600 \text { médicas } \\
\text { ocorridas em } 2008 .\end{array}$ & $\begin{array}{l}\text { A proporção de internações com } \\
\text { pelo menos um EA foi de } \\
12,3 \% \text { e a taxa de incidência } \\
\text { geral do hospital foi de } 14,1 \% \text {. } \\
\text { Aproximadamente metade dos } \\
\text { eventos eram evitáveis. Os EA } \\
\text { foram duas vezes mais } \\
\text { frequentes em pacientes } \\
\text { cirúrgicos. Dos } 82 \text { casos EA } \\
\text { ocorridos nas internações } \\
\text { cirúrgicas, } 74 \% \\
\text { relacionados, majoritariamente, } \\
\text { a falhas técnica e não técnicas, } \\
\text { trombose venosa e infecção da } \\
\text { ferida/sepse pós-operatória. }\end{array}$ \\
\hline
\end{tabular}




\begin{tabular}{|c|c|c|c|c|c|}
\hline $\begin{array}{l}\text { Pérez-Zapata et } \\
\text { al., } \\
\text { 2017, Espanha } \\
\text { Rev. calid. } \\
\text { asist }\end{array}$ & $\begin{array}{l}\text { Comparación } \\
\text { de la } \\
\text { herramienta } \\
\text { Trigger con el } \\
\text { conjunto } \\
\text { mínimo básico } \\
\text { de datos } \\
\text { (CMBD) para } \\
\text { la detección de } \\
\text { eventos en } \\
\text { adversos en } \\
\text { cirugía general }\end{array}$ & $\begin{array}{l}\text { Comparar } \\
\text { desempenho da } \\
\text { ferramenta trigger } \\
\text { na detecção de EA }\end{array}$ & $\begin{array}{l}\text { Retrospectivo, } \\
\text { observacional e } \\
\text { descritivo/VI }\end{array}$ & $\begin{array}{l}125 \text { prontuários de } \\
\text { pacientes submetidos a } \\
\text { cirurgia geral no ano de } \\
2012 \text {. }\end{array}$ & $\begin{array}{l}\text { A prevalência de EA foi de } \\
36,8 \% \text {. Dos } 179 \text { EA detectados, } \\
56,2 \% \quad(\mathrm{n}=101) \text { foram } \\
\text { considerados evitáveis. Infecção } \\
\text { de sítio cirúrgico foi o EA mais } \\
\text { prevalente (24\%), seguido por } \\
\text { fístula }(17 \%) \text { e íleo paralítico } \\
(14 \%) \text {. }\end{array}$ \\
\hline $\begin{array}{l}\text { Araújo et al., } \\
\text { 2018, Brasil. }\end{array}$ & $\begin{array}{l}\text { Eventos } \\
\text { adversos graves } \\
\text { em pacientes } \\
\text { cirúrgicos: } \\
\text { ocorrência e } \\
\text { desfecho }\end{array}$ & $\begin{array}{l}\text { Identificar } \\
\text { ocorrência } \\
\text { eventos adversos } \\
\text { graves (EAG) em } \\
\text { pacientes } \\
\text { cirúrgicos e seus } \\
\text { possíveis } \\
\text { desfechos. }\end{array}$ & $\begin{array}{l}\text { Documental, } \\
\text { retrospectivo, } \\
\text { descritivo- } \\
\text { exploratório, } \\
\text { com abordagem } \\
\text { quantitativa / } \\
\text { VI }\end{array}$ & $\begin{array}{l}\text { Análise de } 19 \text { prontuários } \\
\text { de pacientes cirúrgicos } \\
\text { internados em hospital } \\
\text { privado de São Paulo no } \\
\text { ano de } 2016 \text {. }\end{array}$ & 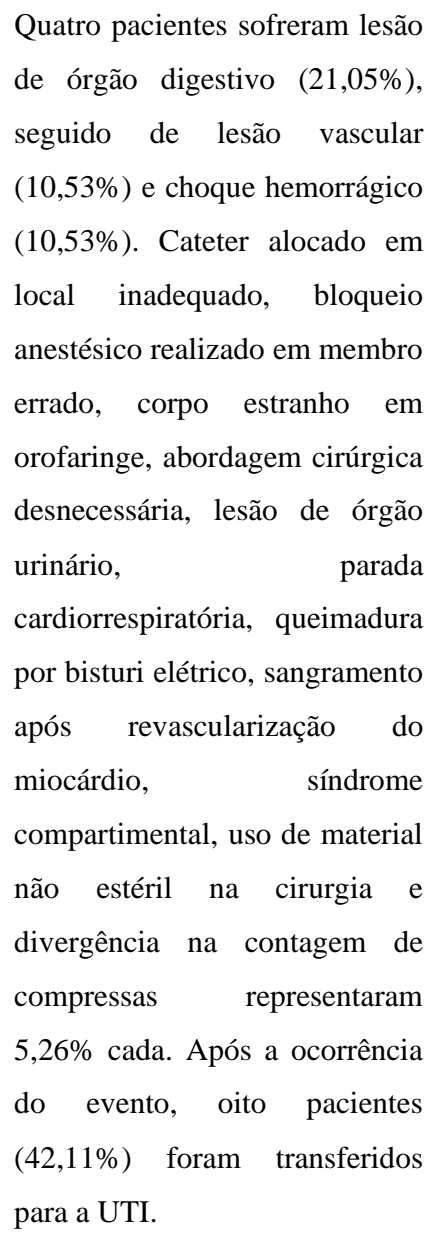 \\
\hline $\begin{array}{l}\text { Chen et al., } \\
2018 \text {, Estudo } \\
\text { Multicêntrico }\end{array}$ & $\begin{array}{l}\text { Improving } \\
\text { detection of } \\
\text { intraoperative } \\
\text { medical errors } \\
\text { (iMEs) and } \\
\text { intraoperative } \\
\text { adverse events } \\
\text { (iAEs) and their } \\
\text { contribution to }\end{array}$ & $\begin{array}{l}\text { Analisar os EA } \\
\text { intra-operatórios }\end{array}$ & $\begin{array}{l}\text { Descritivo e } \\
\text { quantitativo/ VI }\end{array}$ & $\begin{array}{l}\text { Banco de dados com } \\
\text { análise de } 3.020 \text { cirurgias } \\
\text { realizadas no período de } \\
\text { dezembro de } 2015 \text { a } \\
\text { novembro de } 2016 \text {. }\end{array}$ & $\begin{array}{l}\text { Foram encontrados } 103 \text { EA } \\
\text { intraoperatórios. Os eventos } \\
\text { relatados foram hipotensão } \\
(26 \%) \text {, bradicardia }(23 \%) \text { e } \\
\text { hipotermia }(15 \%) \text { Também } \\
\text { foram descritos } \\
\text { decorrentes } \\
\begin{array}{l}\text { perfuração/laceração acidental } \\
\text { de órgãos, hemorragias, reação }\end{array}\end{array}$ \\
\hline
\end{tabular}




\begin{tabular}{|c|c|c|c|c|c|}
\hline & $\begin{array}{l}\text { postoperative } \\
\text { outcomes }\end{array}$ & & & & $\begin{array}{l}\text { alérgica ao látex e erro de } \\
\text { medicação. }\end{array}$ \\
\hline $\begin{array}{l}\text { Sousa et al., } \\
\text { 2018, Portugal } \\
\text { Int J Qual } \\
\text { Health Care }\end{array}$ & $\begin{array}{l}\text { Patient and } \\
\text { hospital } \\
\text { characteristics } \\
\text { that influence } \\
\text { incidence of } \\
\text { adverse events } \\
\text { in acute public } \\
\text { hospitals in } \\
\text { Portugal: a } \\
\text { retrospective } \\
\text { cohort study. }\end{array}$ & $\begin{array}{l}\text { Analisar a variação } \\
\text { na taxa de EA } \\
\text { entre hospitais } \\
\text { agudos e explorar } \\
\text { os fatores que } \\
\text { influenciam nas } \\
\text { diferenças das } \\
\text { taxas de EA }\end{array}$ & $\begin{array}{l}\text { Coorte } \\
\text { retrospectivo/ } \\
\text { IV }\end{array}$ & $\begin{array}{l}\text { Amostra aleatória de } \\
4.225 \text { prontuários de } \\
\text { pacientes internados, no } \\
\text { ano de 2013, em nove } \\
\text { centros hospitalares } \\
\text { públicos. Do total de } \\
\text { prontuários, } 1.866 \text { eram } \\
\text { de pacientes cirúrgicos. }\end{array}$ & $\begin{array}{l}\text { A incidência EA foi de } 12,5 \% \text {; } \\
66,4 \% \text { dos casos estavam } \\
\text { relacionados com infecção } \\
\text { hospitalar e procedimentos } \\
\text { cirúrgicos e cerca de } 40 \% \text { eram } \\
\text { evitáveis. Dos pacientes } \\
\text { cirúrgicos, } 218(11,7 \%) \text { foram } \\
\text { acometidos por EA. }\end{array}$ \\
\hline $\begin{array}{l}\text { Hu et al., } \\
\text { 2019, China. } \\
\text { J Evid Based } \\
\text { Med }\end{array}$ & $\begin{array}{l}\text { Adverse events } \\
\text { identified by the } \\
\text { global trigger } \\
\text { tool at a } \\
\text { university } \\
\text { hospital: A } \\
\text { retrospective } \\
\text { medical record } \\
\text { review }\end{array}$ & $\begin{array}{l}\text { Descrever } \\
\text { prevenção e as } \\
\text { categorias de } \text { EA } \\
\text { em pacientes } \\
\text { geriátricos }\end{array}$ & $\begin{array}{l}\text { Descritivo e } \\
\text { retrospectivo/ } \\
\text { VI }\end{array}$ & $\begin{array}{l}480 \text { prontuários de } \\
\text { pacientes com idade igual } \\
\text { ou superior a } 60 \text { anos } \\
\text { internados no ano de } \\
2015 \text {. }\end{array}$ & $\begin{array}{l}\text { Foram detectados } 610 \text { EA. Dos } \\
249 \text { pacientes cirúrgicos, } \\
78,71 \% \text { apresentaram pelo } \\
\text { menos um EA. Os EA mais } \\
\text { comuns foram os relacionados } \\
\text { aos de origem infecciosa e } \\
\text { medicamentos }(44,17 \%) \text { A } \\
\text { incidência de EA em pacientes } \\
\text { cirúrgicos foi significativamente } \\
\text { maior comparado aos EA } \\
\text { ocorridos em pacientes clínicos } \\
(p<0,001) \text {. }\end{array}$ \\
\hline $\begin{array}{l}\text { Batista et al., } \\
\text { 2019, Brasil. }\end{array}$ & $\begin{array}{l}\text { Prevalência e } \\
\text { prevenção de } \\
\text { eventos } \\
\text { adversos } \\
\text { cirúrgicos em } \\
\text { hospital de } \\
\text { ensino do Brasil }\end{array}$ & $\begin{array}{l}\text { Estimar a } \\
\text { prevalência } \\
\text { prevenção de EA } \\
\text { cirúrgicos em } \\
\text { hospital de ensino } \\
\text { e classificar os } \\
\text { eventos segundo o } \\
\text { tipo de incidente e } \\
\text { grau do dano. }\end{array}$ & $\begin{array}{l}\text { Transversal e } \\
\text { retrospectivo/ } \\
\text { VI }\end{array}$ & $\begin{array}{l}\text { Análise de } \\
\text { prontuários de pacientes } \\
\text { internados no período de } \\
\text { junho de } 2014 \text { a maio de } \\
2015 \text {. }\end{array}$ & $\begin{array}{l}\text { A prevalência de EA cirúrgicos } \\
\text { foi de } 21,8 \% \text {. Dos } 60 \text { casos, } 54 \\
\text { (90\%) foram classificados com } \\
\text { evitáveis. Houve prevalência de } \\
\text { infecção de ferida cirúrgica } \\
\text { (30\%), seguida de deiscência e } \\
\text { hematoma/seroma com 16,7\% e } \\
15 \% \text {, respectivamente. Cerca } \\
\text { de } 40 \% \text { dos casos estiveram } \\
\text { relacionadas a falhas técnicas. }\end{array}$ \\
\hline
\end{tabular}

Legenda: EA - Evento adverso. EAG - Evento adverso grave. Evento adversos a medicamentos - EAM. Estados Unidos da América EUA. Fonte: Autores (2020).

\section{Discussão}

Os resultados dos estudos que compuseram a presente revisão integrativa apontam diferenças distintas entre a incidência/prevalência e prevenção de EA ocorridos em pacientes cirúrgicos, o que corrobora com outra revisão integrativa ao confirmar essas variações entre os estudos e revelar que a qualidade dos registros nos prontuários e do cuidado ofertado, o 
número de critérios de rastreamento utilizados, e a expertise dos revisores primários e secundários contribuem para esses achados heterogêneos (Zanetti et al., 2020).

Constatou-se que os estudos que investigaram a ocorrência de EA em internações gerais apresentaram maior proporção de EA em pacientes cirúrgicos, conforme pesquisa realizada em hospital da Suíça, em que os EA em hospitalizações cirúrgicas foram superiores comparados aos eventos ocorridos em pacientes da clínica médica (Halfon et al., 2017) e de investigação em hospital universitário chinês que identificou incidência de EA em pacientes cirúrgicos significativamente maior do que em pacientes clínicos ( $<<0,001$ ) (Hu et al., 2019). Esses dados reforçam a vulnerabilidade do paciente submetido ao procedimento anestésico-cirúrgico e incitam os serviços de saúde a adotarem ações e práticas seguras, conforme estabelecido pela OMS em segundo desafio global em segurança do paciente “Safe Surgery Saves Lives” (Ribeiro, 2017).

A prevenção dos EA variou de 7,7\% para casos relacionados à medicação (Boer et al., 2013) a 90\% para eventos gerais (Batista et al., 2019). Nesse quesito, cabe destacar que a elevada prevenção dos casos identificados incita adotar estratégias para mitigar os erros para corrigir processos e minimizar os impactos dos EA, haja vista que a maioria são passíveis de prevenção mediante ações institucionais, com destaque para as causas acidentais como perfurações/lacerações.

Destacaram-se os EA de origem infecciosa, por serem os mais frequentes em internações cirúrgicas (Batista et al., 2019) e pelo uso de medicamentos/fluídos endovenosos. Em enfermarias de atendimento ao paciente cirúrgico da Holanda, 73,6\% dos EA estiveram relacionados a medicamentos de uso para o sistema nervoso como os opioides (Boer et al., 2013), enquanto que na série temporal (2010-2011), estudo norte-americano revelou prevalência de eventos relacionados a administração de heparina endovenosa (32\%), seguido de nefropatia após angiografia com contraste (29,3\%) (Wang et al., 2014). Em hospital da região Centro-Oeste do Brasil, 35,5\% dos EA relacionados à assistência foram ocasionados pela anestesia (Souza et al., 2011).

Outro ponto importante é ocorrência dos never events. Esses são eventos que nunca deveriam acontecer, por exemplo, cirurgia no local errado, e são incidentes graves e evitáveis que podem causar danos graves ou até a morte de pacientes (Berner et al., 2020). Investigação realizada por pesquisadores dos Estados Unidos da América (EUA) encontrou 70 casos, com destaque para objetos retidos inadvertidamente e colocação implantes errados, com necessidade de reoperação (Thiels et al., 2015).

Desta forma, torna-se necessário aprimorar condutas que visem prevenir EA associados ao uso de medicamentos de alta vigilância e dos fatores contribuintes para os never events. Sabe-se que os erros humanos e lapsos de memórias estão relacionados a ocorrência de EA anestésicos (Lemos et al., 2019) da mesma forma que o excesso de confiança e distrações (Thiels et al., 2015), e não verificar ou não seguir protocolo contribuem para elevar os riscos assistenciais, incorrendo em EA (Moura et al., 2012).

Observou-se que a taxa de mortalidade encontrada foi de 1,82\% no México (Medina-franco et al., 2006) e estimada em 18,4\% em hospitais situados na região sudeste do Brasil (Moura et al., 2012). A utilização do checklist cirúrgico vem apresentando resultados promissores para reduzir EA, especialmente, os óbitos (Ramsay et al., 2019). No entanto, percebe-se que, em pacientes hospitalizados nos EUA entre 2005 e 2011 por pneumonia e por condições que requerem cirurgia não houve redução significativa nas taxas de EA (Wang et al., 2014).

Sabe-se que o tempo é fator determinante para que mudanças ocorram em indicadores estruturais e de processo bem como possa impactar em melhorias direcionadas a habilidades técnicas e não-técnicas. Somado a esses fatores, destaca-se a relevância da percepção e do comportamento dos profissionais de saúde e de gestores acerca das diversas questões relativas para promoção e adoção de práticas seguras (Batista et al., 2020). Todos esses fatores individuais e/ou coletivos e sistêmicos impactam diretamente nos resultados cirúrgicos e na qualidade da assistência cirúrgica, podendo reduzir os EA e os óbitos nesse importante contexto assistencial. 
Como limitação, destaca-se os diferentes métodos utilizados para busca, identificação e confirmação dos EA nos estudos elegíveis na presente revisão integrativa, o que dificulta comparações e generalizações dos resultados. A inclusão de artigos apenas nos idiomas português, inglês e espanhol disponíveis somente em três bases de dados se somam às limitações.

\section{Considerações Finais}

Neste estudo, foi possível constatar prevalência e prevenção referente aos EA e óbitos ocorridos em internações cirúrgicas. Houve destaque de casos relacionados à infecção e ao uso e administração de medicamentos. Habilidades técnicas e não técnicas contribuíram para a ocorrência dos EA. Destaca-se, de maneira geral, a necessidade de se avançar em ações preventivas para corrigir processos e atenuar a ocorrência de incidentes com danos aos pacientes internados em unidades de atendimento perioperatório.

Espera-se que a presente revisão integrativa possa contribuir para o ensino técnico e superior acerca da segurança do paciente cirúrgico e da realização de novas pesquisas, com vista a desvelar outros diagnósticos situacionais e elencar novas estratégias que possam contribuir para garantir a segurança perioperatória.

\section{Agradecimentos}

Ao Centro Universitário Santa Cruz de Curitiba - UNISANTACRUZ.

\section{Referências}

Agência Nacional de Vigilância Sanitária (2017). Assistência Segura: Uma Reflexão Teórica Aplicada à Prática. https://www20.anvisa.gov.br/segurancadopaciente/index.php/publicacoes/item/caderno-1-assistencia-segura-uma-reflexao-teorica-aplicada-a-pratica

Araújo, S. S. de., Carvalho, R. de. (2018). Serious adverse events in surgical patients: occurrences and outcomes. Revista Sobecc, 23(2): 77-83. https://doi.org/10.5327/Z1414-4425201800020004

Baines, R. J., Langelaan, M., Bruijne, M. C. de, Asscheman, H., Spreeuwenberg, P., Van de Steeg, L., \& Wagner, C. (2013). Changes in adverse event rates in hospitals over time: a longitudinal retrospective patient record review study, BMJ quality \& safety, 22(4), 290-298. http://doi.org/10.1136/bmjqs-2012-001126

Bath, M., Bashford, T., \& Fitzgerald, J. E. (2019). What is 'global surgery'? Defining the multidisciplinary interface between surgery, anaesthesia and public health, BMJ Global Health, 4: e001808. https://gh.bmj.com/content/bmjgh/4/5/e001808.full.pdf

Batista, J., de Almeida Cruz, E. D., Alpendre, F. T., Rocha, D. J. M. da, Brandão, M. B., \& Maziero, E. C. S. (2019). Prevalence and avoidability of surgical adverse events in a teaching hospital in Brazil, Rev. Latino-Am. Enfermagem. 27, e2939. https://doi.org/10.1590/1518-8345.2939.3171

Batista, J., de Almeida Cruz, E. D., Lopez, E. D. C. M. S., Sarquis, L. M. M., Seiffert, L. S., \& Wolff, L. D. G. (2020). Efeito da transição administrativa da gestão hospitalar na cultura de segurança em unidades cirúrgicas. Texto \& Contexto Enfermagem, 29, e20190012. https://doi.org/10.1590/1980-265x-tce-20190012

Berner, J. E., Gokani, V., Savage, J., \& Blackburn, A. (2020). Repeated never events in plastic surgery: Can human factors help us understand why we fail? Journal of plastic, reconstructive \& aesthetic surgery: JPRAS, 73(1), 184-199. https://doi.org/10.1016/j.bjps.2019.05.048

Boer, M. de., Kiewiet, J. J.S., Boeker, E. B., Ramrattan, M. A., Dijkgraaf, M. G. W., Lie-A-Huen, L., \& Boermeester, M. A. (2013). A targeted method for standardized assessment of adverse drug events in surgical patients, Journal of evaluation in clinical practice, 19(6), 1073-1082. https://doi.org/10.1111/jep.12033

Breteler, M. J. M., KleinJan, E., Numan, L., Ruurda., J. P., Hillegersberg, R. V., Leenen, L. P. H., \& Blokhuis, T. J. (2020). Are current wireless monitoring systems capable of detecting adverse events in high-risk surgical patients? A descriptive study, Injury, 51(2), 97-105. https://doi.org/10.1016/j.injury.2019.11.018

Carneiro, F. S., Bezerra, A. L. Q., Silva, A. E. B de C., Souza, L. P de, Paranaguá, T. T. de B., \& Branquinho, N. C. da S. S. (2011). Adverse events in the surgical clinic of a university hospital: a tool for assessing quality, Rev. enferm. UERJ, 19(2), 204-11. http://repositorio.bc.ufg.br/handle/ri/15685

Chen Q., Rosen A. K., Amirfarzan, H., Rochman, A., \& Itani, K. M. F. (2018). Improving detection of intraoperative medical errors (iMEs) and intraoperative adverse events (iAEs) and their contribution to postoperative outcomes, Am J Surg. 216(5), 846-850. https://doi.org/10.1016/j.amjsurg.2018.03.005

Conselho Nacional de Secretários de Saúde - CONASS. (2017). Programa Nacional de Segurança do Paciente (PNSP). http://www.conass.org.br/programanacional-de-seguranca-do-paciente-pnsp/

Galvão, T. F., Pansani, T. de. S. A., \& Harrad, D. (2015). Principais itens para relatar Revisões sistemáticas e Meta-análises: A recomendação PRISMA, Epidemiologia e Serviços de Saúde, 24 (2), 335-342. https://doi.org/10.5123/S1679-49742015000200017 
Halfon, P., Staines. A., Burnand. B. (2017). Adverse events related to hospital care: a retrospective medical records review in a Swiss hospital, International Journal for Quality in Health Care, 29(4), 527-533. https://doi.org/10.1093/intqhc/mzx061

Haynes, A. B., Edmondson, L., Lipsitz, S. R., Molina, G., Neville, B. A., Singer, S. J., \& Berry, W. R. (2017). Mortality trends after a voluntary checklistbased surgical safety collaborative, Annals of surgery, 266(6), 923-929. https://doi.org/10.1097/SLA.0000000000002249

Hu, Q., Wu, B., Zhan, M., Jia, W., Huang, Y., \& Xu, T. (2019). Adverse events identified by the global trigger tool at a university hospital: A retrospective medical record review, J Evid Based Med, 12(2),91-97. https://doi.org/10.1111/jebm.12329

Kohn, L. T., Corrigan, J. M., \& Donaldson, M. S. (2000). To err is human: building a safer health system. Institute of Medicine, National Academy Press, 312 p.

Lemos, C. de Santana., \& de Brito Poveda, V. (2019). Adverse events in anesthesia: An integrative review. Journal of PeriAnesthesia Nursing, 34(5), 978998. https://doi.org/10.1016/j.jopan.2019.02.005

Lopes, M. da. C. da. R, Silva, L. de. F. da., Soares, T., Barros, T. S., Martins, F. J. G., \& Farias, M. S. (2018). Atuação da enfermagem no processo de cirurgia segura, Revista Tendências da Enfermagem Profissional, 10(4), 34-39. http://www.coren-ce.org.br/wp-content/uploads/2020/01/Atua\%C3\%A7\%C3\%A3o-daenfermagem-no-processo-de-cirurgia-segura.pdf

Medina-Franco, H., de la-Medina, A, R., \& Torres-Villalobos, G., León, H. T. C. de. (2006). Factores asociados a mortalidad en cirugía mayor: análisis retrospectivo en un centro de referência, Revista de investigación clínica, 58(1), 9-14. http://www.scielo.org.mx/scielo.php?script=sci_abstract\&pid=S0034$83762006000100002 \& \operatorname{lng}=$ pt\&nrm=iso\&tlng=es

Melnyk, B. M., \& Fineout-Overholt E. (2005). Making the case for evidence-based practice. In: Melnyk BM, Fineout-Overholt E. Evidence based practice in nursing \& healthcare. A guide to best practice. Philadelphia: Lippincott Williams \& Wilkins, p. 3-24.

Mendes, K. D. S., Silveira, R. C. de. C. P., \& Galvão, C. M. (2008). Revisão integrativa: método de pesquisa para a incorporação de evidências na saúde e na enfermagem, Texto \& contexto-enfermagem, 17(4), 758-764. https://doi.org/10.1590/S0104-07072008000400018

Moura, M. de L. de O., \& Mendes, W. (2012). Assessment of surgical adverse events in Rio de Janeiro hospitals, Revista Brasileira de Epidemiologia, 15(3), 523-35. https://doi.org/10.1590/S1415-790X2012000300007

Moura, M. de. L. de. O., \& Santos Diego, L. A. dos. (2017). Lack of access to surgery: a public health problem, Cadernos de Saúde Pública, 33(10), e00151817. https://doi.org/10.1590/0102-311x00151817

Paranaguá; T. T. de B., Bezerra, A. L. Q., Silva, A. E. B. de C., \& Filho, F. M. de A. (2013). Prevalence of no harm incidents and adverse events in a surgical clinic, Acta Paulista de Enfermagem. 26(3), 256-62. https://doi.org/10.1590/S0103-21002013000300009

Pérez-Zapata, A. L., Samaniego, M. G., Cuéllar, E. R., Cámara, A. G. L. de., \& López, P. R. (2017). Comparación de la herramienta Trigger con el conjunto mínimo básico de datos (CMBD) para la detección de eventos adversos en cirugía general, Revista calidad assistencial, 32(4), 209-214. http://dx.doi.org/10.1016/j.cali.2017.01.001

Ramsay, G., Haynes, A. B., Lipsitz, S. R., Solsky, I., Leitch, J., Gawande, A. A., \& Kumar, M. (2019). Reducing surgical mortality in Scotland by use of the WHO Surgical Safety Checklist. British Journal of Surgery, 106(8),1005-11. https://doi.org/10.1002/bjs.11151

Ribeiro, H. C. T. C. (2017). Global patient safety challenges: breve panorama dos avanços e pesquisas no Brasil, Revista de Enfermagem do Centro-Oeste Mineiro, 7. https://doi.org/10.19175/recom.v7i0.2691

Sell, B. T., Amante, L. N., Martins, T., Sell, C. T., Pinho, F. M. de., \& Silva, R. da. (2016). Adverse events in a hospital surgical unit: a descriptive study, Revista SOBECC, 21(3), 146-153. https://doi.org/10.5327/Z1414-4425201600030005

Silva, F. G. da, Oliveira Junior, N. J. de., Oliveira, D. O. de., Nicoletti, D. R., \& Comin, E. (2015). Análise de eventos adversos em um centro cirúrgico ambulatorial, Revista Sobecc, 20(4), 202-209. https://doi.org/10.5327/Z1414-4425202000020003

Sousa, P., Uva, A. S., Serranheira, F., Uva, M. S., \& Nunes, C. (2018). Patient and hospital characteristics that influence incidence of adverse events in acute public hospitals in Portugal: a retrospective cohort study. Int J Qual Health Care. 30(2), 132-137. https://doi.org/10.1093/intqhe/mzx190

Souza, L. P. de, Bezerra, A. L. Q., Silva, A. E. B. de C., Carneiro, F. S., Paranaguá, T. T. de B., \& Lemos, L. F. (2011). Adverse events: instrument for assessing performance of a university hospital surgical center, Rev. enferm. UERJ, 19(1), 127-33. http://repositorio.bc.ufg.br/handle/ri/15684

Thiels, C. A., Lal, T. M., Nienow, J. M., Pasupathy, K. S., Blocker, R. C., Aho, J. M., \& Bingener, J. (2015). Surgical never events and contributing human factors, Surgery, 158(2), 515-521. https://doi.org/10.1016/j.surg.2015.03.053

Ursi, E. S., \& Gavão, C. M. (2006). Perioperative prevention of skin injury: an integrative literature review. Revista latinoamericana de enfermagem, 14(1), 124-131. https://doi.org/10.1590/S0104-11692006000100017

Wang, Y., Eldridge, N., Metersky, M. L., Verzier, N. R., Meehan, T. P., Pandolfi, M. M. \& Battles, J. (2014). National trends in patient safety for four common conditions, 2005-2011, N Engl J Med, 370, 341-351. https://doi.org/10.1056/nejmsa1300991

World Health Organization. (2008). World alliance for patient safety. The second Global Patient safety challenge. Safe surgery saves lives. Geneva: World Health Organization. http://apps.who.int/iris/handle/10665/70080

World Health Organization. (2009). The Conceptual Framework for the International Classification for Patient Safety. http://www.who.int/patientsafety/taxonomy/icps_full_report.pdf 
Research, Society and Development, v. 10, n. 4, e16810413896, 2021

(CC BY 4.0) | ISSN 2525-3409 | DOI: http://dx.doi.org/10.33448/rsd-v10i4.13896

Zanetti, A. C. B., Gabriel, C. S., Dias, B. M., Bernardes, A., Moura, A. A. de, Gabriel, A. B., \& Lima Júnior, A. J. de. (2020). Assessment of the incidence and preventability of adverse events in hospitals: an integrative review, Revista Gaúcha de Enfermagem, 41, e20190364. https://doi.org/10.1590/19831447.2020.20190364 